\title{
Connectivity-Related Properties of Mobile Nodes Obeying the Random Walk and Random Waypoint Mobility Models
}

\author{
L. Hanzo II., S. M. Mostafavi and R. Tafazolli \\ Centre for Communication Systems Research, University of Surrey, Guildford, GU2 7XH, United Kingdom \\ \{L.Hanzo, S.Mostafavi, R. Tafazolli\}@ surrey.ac.uk
}

\begin{abstract}
This paper independently derives the probability of any pair of uniformly-distributed nodes to be within transmission range of each other in a square-shaped area. It then explores, via simulation, some new applications of this expression. The applications are relevant for scenarios where node mobility is governed by the popular random walk or random waypoint mobility models (RWkMM and RWPMM). Under the RWPMM with pausing, at any time, some nodes will be mobile and some stationary. The positions of mobile nodes are drawn from a nonuniform distribution, while a uniform distribution applies to the stationary nodes. In various forms of the RWkMM, the node spatial distribution is uniform in its steady state. The studied applications include calculating the expected node degree and the node isolation probability. Simulation results show that the considered model is able to predict these connectivity-related properties near-perfectly under a paused RWPMM and with all mobility scenarios under the RWk with reflection model. With the RWPMM, the accuracy decreases as the fraction of time the nodes spend moving increases. However, it is still generally better than the simple $\pi r^{2} / A$ disk-covering model, which is often employed for calculating network connectivityrelated properties in ad hoc networks. Further application of the considered methods is exemplified by calculation of an accurate upper bound on the per-node transmission capacity for contention-based networks, when the nodes are uniformly distributed.
\end{abstract}

\section{INTRODUCTION}

A significant portion of mobile ad hoc network (MANET) simulation studies in the last 10 years have employed the random waypoint and random walk mobility models (RWPMM and RWkMM) for simulating node movements. To the best of our knowledge the RWPMM was first introduced in [1]. Various forms of the RWkMM have been in use for many decades [2], albeit in this paper, we focus on the random walk with reflection mobility model (RWkRMM) [3].

Both models initially position nodes randomly according to a uniform distribution in a bordered simulation area of fixed size, most often a square or a rectangle. In this paper we focus on square-shaped areas. The RWkRMM assigns each node a random uniformly distributed speed in the range $\left[V_{\min }, V_{\max }\right] \mathrm{m} / \mathrm{s}$ and a random travel time and direction. Upon traveling for the selected length of time, a node may pause for a random amount of time. It then chooses a new direction, speed and travel time. The RWkRMM differs from the basic RWkMM in that, on reaching the edge of the square or rectangular simulation area, a node is reflected much like a ball bouncing off a wall. This model produces a uniform steady-state node spatial distribution (SSNSD) regardless of the average node speed [3].

By contrast to the RWkMM, under the RWPMM, each node selects a random uniformly-distributed destination point, instead of a direction. Node speeds may be selected from a uniform distribution in the range $\left[V_{\min }, V_{\max }\right] \mathrm{m} / \mathrm{s}$, or a normal distribution. One complication of the RWPMM is that, despite the initial uniform distribution of the nodes, it produces a nonuniform SSNSD when the nodes are mobile [4]. In fact, since each node's destination point is uniformly-distributed, if all nodes are allowed to reach their destinations before any node moves again, the uniform distribution is re-attained. However, since each node is likely to select a destination on the opposite side of the simulation area, during mobile periods, most nodes are more likely to be found near the centre of the area.

This interesting SSNSD, together with the widespread use of this model for ad hoc network-layer research, prompted analysis of the RWPMM. Expressions for the steady-state probability density function (PDF) of the node positions have been derived for both square and circular simulation areas [4]. The steady-state distributions of node location, speed and pause time, derived in [5], allowed the authors to develop a RWPMM implementation which ensures that node positions and movement speeds are sampled from the steady-state distributions immediately.

Expressions have also been derived for the critical transmission range required for a RWPMM-governed network to be 1-connected, where each node has at least one neighbour [6], and k-connected [7]. The average period of maintaining connectivity was also studied [7]. Furthermore, the expected number of neighbours of a node, i.e. the node degree of a network in a circular simulation area has been derived in [8]. The PDF of the distance moved by a node and of the time of travel before changing direction, as well as the distribution of the angle of movement have been analysed in [9]. Finally, the chance of any pair of nodes to be within transmission range of each other was analysed in [10].

Some of the connectivity-related properties of uniformlydistributed nodes have also been studied (see [10] and references therein). However, to this day, many researchers continue to employ the simple and intuitive disk-covering approach for calculating connectivity-related properties (e.g. 
[11]). In this model, the chance that a node is a neighbour of another is simply $\pi r^{2} / A$, where $r$ is the transmission range and $A$ is the area's size. Indeed, as our results for a square simulation area show, this model is accurate where the transmission range is relatively small (approximately less than $\sqrt{A} / 5$ in our simulations). As the transmission range increases, the accuracy of this model decreases drastically. It was only relatively recently, that a more accurate formula for the node pair connectivity probability was published [10].

These analytical results have deepened understanding of the effect of the considered mobility models on the nature and degree of node mobility, as well as the resulting network connectivity properties. However, as we show in this paper, further applications of the results are possible. Furthermore, with models such as the RWPMM, where the resulting node spatial distribution differs depending on the fraction of time nodes spend paused, it is not always clear which analytical model should be applied for predicting network properties.

Analysis of the connectivity-related properties produced by the considered mobility models is important for several reasons. For example, simulation results can be verified by comparison to the expected results. Where an analytical model is available, time spent on simulations may be used elsewhere. Even if simulation is necessary, time may be saved by discarding parameter combinations that would not produce protocol performance in the useful region.

The main body of this paper begins in Section II by showing an alternative path to calculating the probability of uniformlydistributed nodes to be within transmission range of each other in a square-shaped area. The application of this result to calculating the expected node degree and the node isolation probability is then presented. The analytical expressions are verified through comparisons to simulation results in Section III. Next, Section IV demonstrates how the introduced methods can be used to calculate specific upper bounds on the per-node transmission capacity of a network employing a contentionbased medium access control (MAC) protocol. Conclusions are given in Section V.

\section{NODE CONNECTIVITY}

With the RWPMM, the distance moved by a node between two destination points is often called the transition length [9]. As mentioned above, the coordinates of each new node destination point are random variables with values uniformly distributed over the simulated area. Thus, the same probability distribution applies to each destination point of each node. It thus follows that, once the nodes have stopped moving, the expected transition length is equivalent to the expected distance between two nodes. In a network with a mixture of paused and mobile nodes, the overall node spatial distribution is thus a mixture of a uniform one and the non-uniform one given in [4].

The joint probability density function (PDF) of the two dimensions of the transition length for a square area was reported in [9]. This then equates to the PDF of the distance between two paused nodes. In the following analysis we assume that mobile nodes move in a square area of size $s^{2}$ metres $^{2}$, select speeds uniformly distributed in the range $\left[V_{\min }, V_{\max }\right]$, and have a common transmission range of $r$ metres. Continuing from Equation (18) in [9], we have:

$P(D<L)=\int_{0}^{L} \int_{0}^{\sqrt{L^{2}-x^{2}}} \frac{4}{s^{4}}\left(x y-s(x+y)+s^{2}\right) d x d y$,

where $x$ and $y$ are the distances between two nodes in the $\mathrm{x}$ and y dimensions respectively, $D$ is the euclidean distance between two nodes, and $L$ is a parameter representing a distance of interest, e.g. the transmission range. Converting (1) to the polar domain by $x=r \cos (\theta), y=r \sin (\theta)$, allows us to deal with the circular transmission coverage area. Evaluating the integrals between the new polar domain limits yields the probability $P(D<L)$. We differentiate this expression to acquire the distribution of the node separation distance:

$$
\frac{d P}{d L}=\frac{2 \pi}{s^{2}} L-\frac{8}{s^{3}} L^{2}+\frac{2}{s^{4}} L^{3} .
$$

Finally, to calculate the probability $p_{r}$ that any two nodes are within the transmission range $r$ of each other in a squareshaped simulation area, we integrate the above distribution between the limits $[0, r]$ to yield:

$$
p_{r}=\pi\left(\frac{r}{s}\right)^{2}-\frac{8}{3}\left(\frac{r}{s}\right)^{3}+\frac{1}{2}\left(\frac{r}{s}\right)^{4} .
$$

The same formula was derived differently and independently in [10]. Clearly, the chance for the nodes to be out of range and the link to be broken is then $1-p_{r}$. Note that the previous statement adopts the common assumption that if two nodes are within a distance of $r$ of each other, the signal-to-interferencenoise ratio is sufficiently high at the receiver to enable reliable packet reception. If the nodes are further than $r$ apart, then no direct communications are deemed possible.

Let us now consider a 'link' between two nodes. The link may break if either of the nodes moves. From each node's perspective we can assume that the failure of any of its links is caused by movement of the node at the other end of the link. Thus, the failure events of each link are independent. In this case, the probability $p_{n o n b r}$ of a node having no neighbours in an $n$-node network is simply:

$$
p_{n o n b r}=\left(1-p_{r}\right)^{n-1},
$$

i.e. all of its potential links are broken. We can also apply a similar argument for calculating the expected number of neighbours $E\left[n_{n b}\right]$ of each node:

$$
\begin{gathered}
E\left[n_{n b}\right]=p_{r}(n-1) . \\
\text { III. VALIDATION }
\end{gathered}
$$

In order to validate the results presented in the previous section, we employed the popular ns-2 simulator [12]. Note that no verification of (3) by simulation was performed in [10]. The steady-state version of the RWPMM [5] (implemented 
Table I

SIMULATION PARAMETERS EMPLOYED

\begin{tabular}{|l|l||r|r|}
\hline Parameter & Value & Parameter & Value \\
\hline \hline Simulation area & $500 \mathrm{~m} \times 500 \mathrm{~m}$ & $V_{\max }$ & $\{2,15\} \mathrm{m} / \mathrm{s}$ \\
\hline Simulation time & $500 \mathrm{~s}$ & $V_{\min }$ & $\{1,5\} \mathrm{m} / \mathrm{s}$ \\
\hline Number of nodes & 50 & Transmission range & $10 \mathrm{~m}-500 \mathrm{~m}$ \\
\hline
\end{tabular}

as setdest version 2 in ns-2) was employed in all RWPMMemploying mobile scenarios, in order to ensure that node positions and speeds were sampled from the steady-state distributions immediately. Two speed ranges were studied, 1$2 \mathrm{~m} / \mathrm{s}$ corresponding to pedestrian speeds, and $5-15 \mathrm{~m} / \mathrm{s}$ corresponding approximately to urban vehicle speeds. Table I summarises the main simulation parameters.

Each node's position was traced every second. From this, the number of node pairs in range of each other and the number of isolated nodes were calculated for various values of the transmission range. Finally, these values were averaged over the simulation time and then over all simulation runs. This yielded the chance for a link to be intact/broken, the expected number of neighbours (node degree) and the node isolation probability. Each simulation was run with 50 different mobility scenarios (specifying initial node positions, and the sequence of node movements).

The results for the predicted and simulated node degrees are shown in Figure 1. Note that, due to the direct relationship between the node pair connection probability (3) and the expected node degree (5), the level of accuracy of (5) directly reflects that of (3). Therefore, there is no need to show both graphs.

Let us now examine Figure 1. Clearly, for the RWkRMM, even when the nodes are continuously mobile, (5) exactly predicts a node's average number of neighbours. This is also true, as expected, for the RWPMM for any period where all nodes are allowed to pause after moving, as shown by the data points labeled "RWP-Paused". It is also clear that the simple disk-covering approach becomes less appropriate as the transmission range increases past approximately a fifth of the length of the simulated area. This is because, as $r$ increases, a decreasing portion of nodes' transmission coverage areas fit completely inside the simulated area.

The remaining four data sets verify the expectation that, for the RWPMM, the accuracy of both (3) and (5) decreases when the fraction of time the nodes spend moving increases. This is because the expected node degree increases when the nodes are more likely to be clustered together near the centre of the area. Where the pause time after each movement is 100 s, compared to the 500 s simulation time, the accuracy is still pretty good. The accuracy for pedestrian speeds with $100 \mathrm{~s}$ pause time is worse than for vehicle speeds with 20s pause time because the slower-moving nodes take much longer to reach the end of each of their transitions. However, for all levels of mobility, the proposed method is still more accurate than the disk-covering approach at higher transmission ranges compared to the area length. Figure 1 also verifies that node

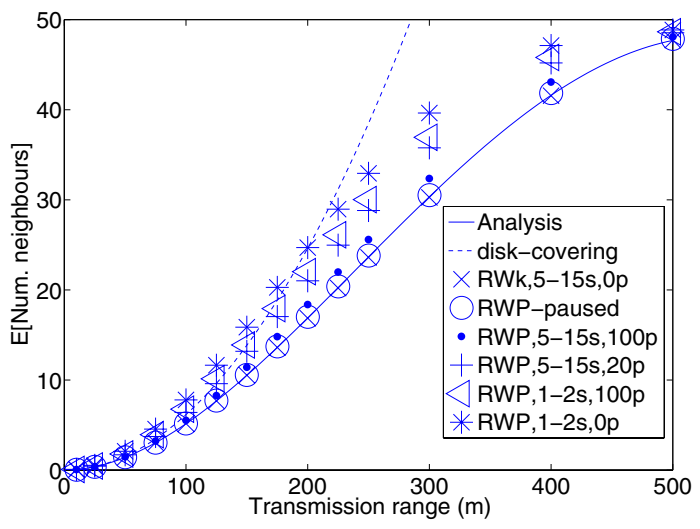

Figure 1. The expected number of neighbours of each node i.e. node degree vs. the common node transmission range. The curve labeled "Analysis" corresponds to the prediction using (5). The curve labeled "disk-covering" corresponds to the simple prediction offered by $n \pi r^{2} / A$, where $A$ is the size of the simulated area. Respectively, RWP and RWk stand for the random waypoint and random walk with reflection mobility models. Further terms in the labels appear in the form " $V_{\min }-V_{\max } \mathrm{s}$, \{pause_time\}p". Simulation parameters are summarised in Table I.

pair connection probabilities are independent of each other, and thus the assumptions behind (5) are valid.

Secondly, Figure 2 presents the analytical and simulation results for the node isolation probability. Again, for the RWPMM, the prediction's accuracy decreases as the fraction of time nodes spend moving increases, and a smaller portion of them are positioned according to a uniform distribution. This time, the approximation provided by the disk-covering model is sufficiently accurate up to the transmission range where it predicts $p_{r}$ to exceed 1, which cannot be seen on Figure 2 . This accuracy is thanks to the fact that the region of interest for node isolation probability is in the accurate region for the diskcovering model (refer to Figure 1). However, in the case of the paused RWP and RWkR mobility models, the disk-covering method is still slightly less accurate than the proposed one above $50 \mathrm{~m}$ transmission range.

Under the RWPMM, the formula for the node pair connection probability given in [10] could easily be combined with (4) and (5) to more accurately calculate the relevant properties when the nodes spend a large fraction of the time moving.

\section{PER-NODE CAPACITY PREDICTION}

In carrier-sense multiple-access with collision avoidance (CSMA/CA)-based MAC protocols, such as the widelyadopted 802.11 distributed coordination function (DCF) [13], a node may only transmit when it senses that the channel is idle. Therefore, the amount of channel time available to a node depends on the traffic at all of the nodes in its carriersensing (cs-) range. The reciprocal of the fraction of the area covered by a node's cs-range gives an estimation for the spatial reuse factor (SRF) of the network. The SRF represents the number of simultaneous transmissions that may occur within a CSMA/CA-based network (see, for example, [14]). Again, 


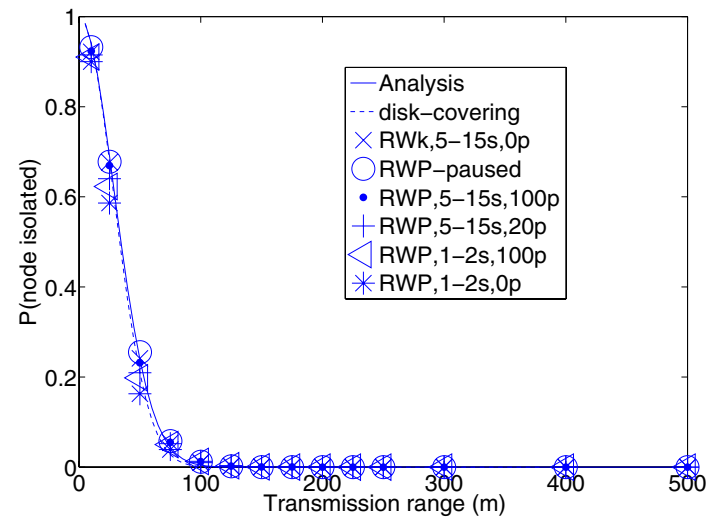

Figure 2. The probability of a node being isolated vs. the common node transmission range. The curve labeled "Analysis" corresponds to the prediction using (4). The other data sets are labeled as explained in the caption for Figure 1.

the disk-covering approach is often adopted for calculating the $\mathrm{SRF}$, as it is in [14].

In communications protocols for multi-hop MANETs, the cs-range is often assumed to be twice the transmission range (e.g. [15]). Employing the same model, (3) can be used to calculate the chance of a node being in another's cs-range $p_{c s r}$. The ratio of the chance for the node to be in the network (i.e. $1)$, to $p_{c s r}$, also yields an estimate for the SRF as:

$$
S R F=\frac{1}{p_{c s r}},
$$

using (3) to calculate $p_{c s r}$. Note that the SRF may also be thought of as the ratio of the total number of nodes to those within the cs-range $n_{c s n b}$, i.e. $S R F=\frac{n}{n_{c s n b}}$, where $n_{c s n b}$ is obtained using (5) with $p_{c s r}$. The transport capacity of the network, and the per-node transmission capacity $C_{n}$ are then simply $C \cdot S R F$ and

$$
C_{n}=\frac{(C \cdot S R F)}{n},
$$

respectively, where $C$ is the channel capacity or node transmission rate. For networks of multi-rate-capable nodes, obviously, the SRF should just be used to provide an estimate of the average fraction of channel time available to a node, instead.

The work in [14] is an example of the disk-covering approach being used, with RWPMM-governed nodes, to predict the SRF and hence the network capacity. We compare this approach, for uniformly-distributed nodes (as in the paused RWPMM or the RWkRMM), to the method of predicting the per-node transmission capacity using (7).

As in Section III, we employed simulations of 50-node networks in a 500mx500m area for verifying (7). However, this time, each node was also a traffic source. We employed the two-ray ground propagation model, although introducing fading would theoretically still produce the same average transmission range over a period of time. Also, we set $2 \mathrm{Mbps}$ as the fixed channel capacity $C$. Using the above method

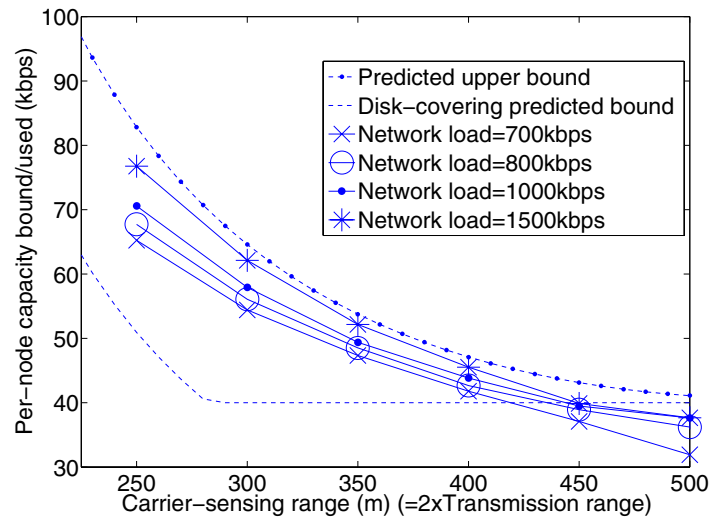

Figure 3. The per-node transmission capacity predicted by our formula and by the disk-covering approach employed in many papers, and achieved by stationary nodes in our simulations with various network loads and transmission and carrier-sensing ranges. The simulations employed 50 nodes in a $500 \mathrm{~m} \times 500 \mathrm{~m}$ area.

for calculating the network capacity as $C \cdot S R F$, we then divided this by the average route length (as in [14]), to yield an estimate of the end-to-end capacity of the network. This estimate lay between $700 \mathrm{kbps}$ and $850 \mathrm{kbps}$, depending on the transmission/cs-range. It was used as a guide on the network traffic load to offer. Simulations were run with $700 \mathrm{kbps}$, $800 \mathrm{kbps}, 1000 \mathrm{kbps}$ and $1500 \mathrm{kbps}$ of total traffic load, which was split equally between the 50 source nodes. The sources began transmitting, one after the other, at two-second intervals. Once they were all transmitting, the measuring of per-node transmission capacity began. We counted all bits transmitted by a node, including data, packet headers and MAC control frames. The average transmission rate was measured over a 50 -second period.

The transmission range and the cs-range were also varied (via the receiving- and cs-thresholds), with the latter being twice the former. Nodes remained stationary with a uniform spatial distribution to ensure that route failures did not prevent the network from operating near its capacity, and that (6) and (7) could be applied with hope of accuracy. Each simulation was run ten times with different node positions, and the average results were collected.

Figure 3 shows the simulation results of per-node capacity compared to the upper bounds predicted by our model and the disk-covering model. As the figure shows, at around $280 \mathrm{~m}$ cs-range, there is a cut-off point in the disk-covering model. This was inserted because at this point the model predicts that the SRF is 1, and since at least one transmission is always possible, the SRF cannot be allowed to drop below 1 .

The results are shown for cs-ranges between $250 \mathrm{~m}$ and $500 \mathrm{~m}$, corresponding to transmission ranges between $125 \mathrm{~m}$ and $250 \mathrm{~m}$. The reason for this range was that, with this node density, network partitioning is very likely below $125 \mathrm{~m}$ transmission range. Also, above 500m cs-range, the SRF does not change significantly in the $500 \times 500 \mathrm{~m}$ area studied.

As can be anticipated after seeing Figure 1, the studied transmission/cs-range region, while being a useful region to 
study, is not in the accurate region for prediction using the disk-covering model. On the other hand, the prediction using (6) and (7) seems to yield an accurate upper bound on the per-node transmission capacity. The upper bound cannot be reached by the 802.11 MAC protocol because of back-off period increases due to collisions. At the lowest simulated load, 700kbps, the average per-node transmission rate was between $65 \mathrm{kbps}$ and $32 \mathrm{kbps}$, depending on the cs-range. As the load was increased, the achieved transmission rate crept towards the predicted upper bound. When the load was more than doubled to $1500 \mathrm{kbps}$, the achieved per-node transmission rate climbed to between $77 \mathrm{kbps}$ and $38 \mathrm{kbps}$. This is a small percentage increase (20\%) compared to the increase in load $(114 \%)$, indicating that the predicted curve does indeed represent an upper bound on the per-node transmission capacity. Note that, at the highest load, depending on the cs-range, a high percentage of packets were lost due to congestion, which also indicates that it is very difficult to achieve a higher endto-end throughput.

When using the RWkRMM, the predicted upper bound would hold since the uniform SSNSD is upheld at all times. However, for mobile nodes, the expected achieved transmission rate would be further below the bound due to pauses in transmissions caused by route failures. For the RWPMM, this predicted bound would be relatively accurate for high pause times and relatively high node speeds, as indicated by Figure 1. At lower speeds and pause times, RWPMM-governed nodes would experience a higher node degree (Figure 1), and hence achieve an even lower per-node transmission rate. Therefore, again, the predicted upper bound would hold, albeit less closely. A more accurate bound may be calculated for this case by applying the node pair connectivity probability formula for the RWPMM from [10] to (6) and (7).

\section{CONCLUSIONS}

This paper independently derived and then employed simulation results to verify a previously-derived analytical expression for the chance of two randomly-positioned nodes to be connected in a square-shaped area. Some new applications of this expression were then demonstrated, starting with the calculation of the expected node degree and the node isolation probability. Again, simulation results verify that the accuracy of these expressions is near-perfect when all node positions are drawn from a uniform distribution. This is true for the RWPMM when all nodes are paused, and for any mobility model which produces a uniform steady state node spatial distribution (SSNSD). Examples of the latter are the random walk with reflection and random walk with wrap-around mobility models (RWkRMM and RWWAMM) [3]. For the RWPMM, the accuracy of the expressions decreases as the average fraction of time nodes are mobile increases. However, in most cases, the methods presented are still more accurate than the predictions offered by the simple $\pi r^{2} / A$ (disk-covering) model that researchers often use (e.g. [11], [14]) to estimate the chance of a node to be within another's transmission range $r$ in an area $A$, and hence other connectivity-related properties.
Further applications of the presented methods are also possible as exemplified in this paper by calculating an upperbound on a network's achievable per-node transmission rate. The predicted bound was sufficiently accurate for stationary nodes and is expected to hold for mobile nodes, albeit less accurately. The above statements regarding the accuracy of the proposed models apply also to the capacity bound estimation.

All of the methods presented in this paper have practical value in verifying simulation results, predicting the suitability of various simulation scenarios for their intended purposes, and saving simulation time by avoiding less-than-useful scenario configurations. The methods were presented and verified largely in the context of uniformly-distributed nodes and they are most accurate in conjunction with the RWkRMM, RWWAMM and the paused RWPMM. However, employing the relevant node pair connection probability expression from [10], the methods can also be applied to continuously-mobile RWPMM-governed nodes. The value of the fraction of time nodes spend moving, at which that other expression would yield more accurate results under the RWPMM, has not yet been determined.

\section{REFERENCES}

[1] D. B. Johnson and D. A. Maltz, "Dynamic source routing in ad hoc wireless networks," in Mobile Computing, Imielinski and Korth, Eds. Kluwer Academic Publishers, 1996, vol. 353.

[2] T. Camp, J. Boleng, and V. Davies, "A survey of mobility models for ad hoc network research," Wireless Communications and Mobile Computing, vol. 2, no. 5, pp. 483-502, 2002.

[3] J. Le Boudec and M. Vojnovic, "Perfect simulation and stationarity of a class of mobility models," in Proc. IEEE INFOCOM, vol. 5, 2005, pp. 72-79.

[4] C. Bettstetter, G. Resta, and P. Santi, "The node distribution of the random waypoint mobility model for wireless ad hoc networks," IEEE Trans. Mobile Comput., vol. 2, no. 3, pp. 257-269, July 2003.

[5] W. Navidi and T. Camp, "Stationary distributions for the random waypoint mobility model," IEEE Trans. Mobile Comput., vol. 3, no. 1, pp. 99-108, Jan. 2004.

[6] P. Santi, "The Critical Transmission Range for Connectivity in Mobile Ad hoc Networks," IEEE Trans. Mobile Comput., vol. 4, no. 3, pp. 310-317, May. 2005.

[7] P. Lassila, E. Hyytiä, and H. Koskinen, "Connectivity Properties of Random Waypoint Mobility Model for Ad Hoc Networks," in Proc. 4th Mediterranean Wksp. Ad Hoc Networks, Île de Porquerolles, France, Jun. 2005, pp. 159-168.

[8] C. Bettstetter, "On the connectivity of ad hoc networks," The Computer Journal, vol. 47, no. 4, pp. 432-447, 2004.

[9] C. Bettstetter, H. Hartenstein, and X. Pérez-Costa, "Stochastic properties of the random waypoint mobility model," ACM Wireless Networks, vol. 10, no. 5, pp. 555-567, Sep. 2004.

[10] G. Farhadi and N. Beaulieu, "Connectivity and Bit Error Rate Analysis of Mobile Ad Hoc Wireless Networks," in Proc. 64th Vehicular Technology Conf., Fall, 2006, pp. 1-5.

[11] E. Miorando and F. Granelli, "On Connectivity and Capacity of Wireless Mesh Networks," in Proc. IEEE Int. Conf. Communications (ICC), 2007, pp. 91-95.

[12] K. Fall and K. Varadhan(eds.), "The ns manual," Available online: http: //nsnam.isi.edu/nsnam/index.php/Main_Page.

[13] IEEE Computer Society, Wireless LAN Medium Access Control (MAC) and Physical Layer (PHY) Specifications, 2007, IEEE Std. 802.11-2007.

[14] R. Zhang and I. Rubin, "Robust Flow Admission Control and Routing for Mobile Ad hoc Networks," in Proc. Military Communications Conference (MILCOM), Los Angeles, CA, USA, Oct. 2006, pp. 1-7.

[15] Y. Yang and R. Kravets, "Contention-aware admission control for ad hoc networks," IEEE Trans. Mobile Comput., vol. 4, no. 4, pp. 363-377, Aug 2005 . 